\title{
Evolution of pulse shapes during compressor scans in a CPA system and control of electron acceleration in plasmas
}

\author{
Csaba Tóth, Joeri de Groot, Jeroen van Tilborg, Cameron G. R. Geddes, \\ Jerome Faure, Palma Catravas, Carl Schroeder, B. A. Shadwick, Eric Esarey, and \\ Wim Leemans
}

\author{
L'OASIS Group, Accelerator and Fusion Research Division Lawrence Berkeley \\ National Laboratory, MS 71-259, 1 Cyclotron Rd., Berkeley, CA 94720, USA \\ E-mail: ctoth@lbl.gov
}

\begin{abstract}
The skewness of the envelope function of 50-100 femtosecond Ti:sapphire laser pulses has been controlled by appropriate choice of the higher order spectral phase coefficients, and used for optimization of a plasma wake-field electron accelerator.
\end{abstract}

\section{Introduction}

The delicate details of the envelope function of ultrashort light pulses ('pulse shape') play an important role in most nonlinear processes. Two pulses with the same full width half maximum (FWHM), but having different rising edge and tail characteristics or amplitude modulation, could lead to strongly different results, especially in cases where certain cumulative processes are involved in the first part of the interactions. For example, in laser wake-field acceleration [1], a driving pulse with a sharp leading edge will result in faster growth of the plasma wakefield, and consequently, higher energy electrons after acceleration.

There are several means of active control of the shape of ultrashort pulses, such as frequency-domain filtering and envelope transfer to the time-domain by using liquid crystal phase modulators [2] or acousto-optic phase and amplitude control [3]. In this report we investigate the intrinsic pulse shaping behavior of the most widely used optical pulse compressor, the grating pair - with special emphasis on the pulse skewness and its effect on a strongly nonlinear process, the laser wakefield generation.

\section{Envelopes, chirp and skew}

The properties of an ultrashort pulse depend on the higher order phase characteristics. In addition to the simplest case of linear 'chirp' (when the phase of an optical pulse changes quadratically as a function of time or frequency), the presence of higher order components (such as 'cubic' third order, or 'quartic' fourth order) will modify the shape of the pulse, resulting in modulation, and even pre- or post pulses $[4,5]$. In every chirped pulse amplification (CPA) system, the final 
pulse-forming device is the grating pulse compressor. In contrast to the most simplistic view and practice, by scanning the grating separation the experimenter changes not only the linear chirp, but also modifies all the higher order terms [6]. This effect becomes negligible far from the shortest compression settings, i.e., where the pulse duration is much longer than the shortest possible. However, if the pulse duration is in the range of a few times the shortest one, or the compressor angle is set slightly differently from the optimum, or the pulse contains uncompensated higher order terms, then a typical scan around the shortest pulse will produce significant changes to the pulse shape. For example, when compressing stretched pulses with a positive bias third order phase component, the pulse shape in the course of a compressor separation scan initially will be skewed toward the head of the pulse $(s<1)$, then flips to become a skewed pulse at the tail $(s>1)$, and again flips back to the original asymmetric one $(s<1)$. The skewness parameter (s) is conveniently defined here as the ratio of the 'head-width-half-max' (HWHM) and the 'tail-width-half-max' (TWHM), respectively. We note here, that these changes are fundamentally different from the well known and widely used mapping of the spectral amplitude to the time domain at strongly chirped pulses, where only the dominant, second order phase coefficient plays any role.

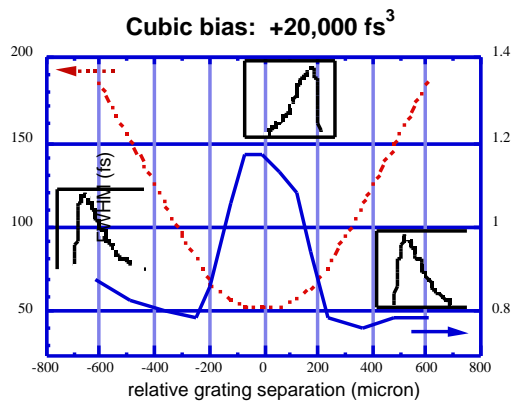

(a)

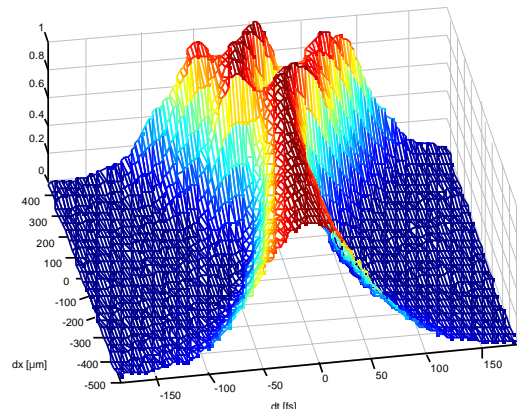

(b)

Fig. 1. The variation of skewness and pulse width (a), and global shape of the envelope (b), in the function of grating separation.

The described qualitative behavior can be clearly seen in Figure 1(a), which shows the pulse shape dependence of a $\sim 19 \mathrm{~nm}$ bandwidth pulse during an experimental compressor scan around the shortest pulse. On the small inserts time flows from left to right. The grating parameters are: groove density: $14801 / \mathrm{mm}$; angle of incidence: $50.0 \mathrm{deg}$; grating separation at the shortest pulse: $323 \mathrm{~mm}$; l=795 nm; third order bias: $+20,000 \mathrm{fs}^{3}$. In addition to the conventional autocorrelation measurement - which usually washes out the details of the pulse shape - we measured the real pulse envelope by using a PG-FROG device [8], showing the same flip in characteristics of pulse skewness around the shortest pulse. A more detailed simulation of the pulse shape evolution using the experimentally retrieved parameters of the pulse can be seen on Figure 1(b). On the time-axis time flows from the right to the left, and the front region of the plot 
represents the positive chirp cases, while the back corresponds to negatively chirped pulses.

\section{Option for control of wakefield accelerators}

In laser plasma wake-field electron acceleration experiments in the L'OASIS Lab of LBNL we observed strong asymmetry in the yield of neutrons produced by accelerated electrons of multiple-tens of $\mathrm{MeV}$ as a function of compressor scans $[8,9]$. There is a strong indication that the interplay of pulse shapes and sign of chirp leads to enhanced growth of the plasma wake in the standard wake-field accelerator regime at early parts of the interaction. This process eventually results in optimal acceleration conditions different from the shortest, highest intensity pulse, favoring pulse shapes with sharp leading edges. The detailed knowledge of the evolution of the pulse shapes provides us an additional path to control the parameters of plasma wake-field accelerators even in a conventional grating compressor scan by intentionally biasing the higher order phase components. The presented method of higher order phase control is a simple alternative to the recently developed acousto-optic phase modulation devices such as the DAZZLER [3], and can conveniently be used at arbitrarily high power and energy levels limited only by the damage threshold of the compressor gratings.

Acknowledgements. This work has been supported by the DOE contract DEAC-03-76SF0098. C. Geddes is also supported by the Hertz Foundation.

\section{References}

1 W. P. Leemans, C. W. Siders, E. Esarey, N. E. Andreev, G. Shvets, and W. B. Mori, IEEE Transactions on Plasma Science, 24, 331-342, 1996.

2 A. M. Weiner, D. E. Leaird, J. S. Patel, and J. R. Wullert, IEEE Journal of Quantum Electronics, 2 8, 908-920, 1992.

3 F. Verluise, V. Laude, Z. Cheng, C. Spielmann, and P. Tournois, Optics Letters, $25,575-577,2000$.

4 D. N. Fittinghoff, B. C. Walker, J. A. Squier, Cs. Tóth, C. Rose-Petruck, and C. P. J. Barty, IEEE J. Sel. Top. Quant. Elec. JSTQE-4, 430-440, 1998.

5 S. Backus, C. G. Durfee, III, M. M. Murnane, and H. C. Kapteyn, Rev. Sci. Inst. 69, 1207-1223, 1998.

6 Cs. Tóth, D. N. Fittinghoff, B. C. Walker, J. A. Squier, C. P. J. Barty, in Ultrafast Phenomena XI, Edited by T. Elsaesser, J. G. Fujimoto, D. A. Wiersma, W. Zinth (Springer, Berlin, 1998) pp. 109-111.

7 D. J. Kane and R. Trebino, Optics Letters, 18, 823-825, 1993.

8 W. P. Leemans, D. Rodgers, P. E. Catravas, C. G. R. Geddes, G. Fubiani, E. Esarey, B. A. Shadwick, R. Donahue, and A. Smith, Physics of Plasmas, 8, 2510-2516, 2001.

9 W. P. Leemans, P. Catravas, E. Esarey, C.G.R. Geddes, C. Tóth, R. Trines, C. B. Schroeder, B. A. Shadwick, J. van Tilborg, and J. Faure, submitted to Phys. Rev. Lett., 2002. 